\title{
Effects of Meteorological Factors on Yield Characters of Waxy Corn at Different Sowing Dates
}

\author{
Heping Tan, Fucheng Zhao, Hailiang Han, Fei Bao, Bin Chen, Junfeng Hou, Xiaocheng Lou, \\ Guiyue Wang*
}

Institute of Maize and Featured Upland Crops, Zhejiang Academy of Agicultural Sciences, Dongyang, P. R. China

Email address:

heping_tan@163.com (Heping Tan), encliff@163.com (Fucheng Zhao), hhl522@163.com (Hailiang Han), baofei8312@126.com (Fei Bao), 414841168@qq.com (Bin Chen),1604622263@163.com (Junfeng Hou),dylxc@163.com (Xiaocheng Lou),

zjdygy@163.com (Guiyue Wang)

${ }^{*}$ Corresponding author

\section{To cite this article:}

Heping Tan, Fucheng Zhao, Hailiang Han, Fei Bao, Bin Chen, Junfeng Hou, Xiaocheng Lou, Guiyue Wang. Effects of Meteorological Factors on Yield Characters of Waxy Corn at Different Sowing Dates. Journal of Plant Sciences. Vol. 8, No. 6, 2020, pp. 214-220.

doi: $10.11648 /$ j.jps.20200806.14

Received: December 3, 2020; Accepted: December 14, 2020; Published: December 28, 2020

\begin{abstract}
In production, the yield of waxy corn is often improved by adjusting fertilization and planting density, while the influence of metecological factors on yield is ignored. In this study, the change of waxy corn growth stage and the influence of main meteorological factors on the yield of fresh ear at the best harvest time were analyzed by adjusting the sowing date. The results showed that the duration of each growth stage was shortened with the delay of sowing date. Correlation analysis showed that daily temperature difference, rainfall, sunshine duration and effective accumulated temperature in the filling stage had significant correlation with most yield characters. The daily temperature difference, sunshine duration and effective accumulated temperature were significantly positively correlated with ear diameter, grain number per row, 100-grain weight and fresh ear yield, but negatively correlated with bald tip length. Combined with regression analysis, the fresh ear yield had a significant quadratic curve relationship with the main meteorological factors in the filling stage. The fresh ear yield had an increasing relationship with the daily temperature difference, sunshine duration and effective accumulated temperature in the filling stage, and a decreasing relationship with the rainfall, and an increase and then decrease relationship with the daily average temperature. The results showed that hot and rainy during filling stage of spring waxy corn in central Zhejiang Province was not conducive to the increase of fresh ear yield, commodity and 100-grain weight. It was suggested that early sowing was beneficial to weaken the influence of unfavorable meteorological factors during filling stage.
\end{abstract}

Keywords: Waxy Corn, Sowing Dates, Meteorological Factors, Fresh Ear Yield, Correlation

\section{Introduction}

The increase of corn yield is not only affected by the genotypes of the variety itself, but also directly related to climatic conditions. Thus, it is of great significance to study the relationship between meteorological factors such as light, temperature, water and yield performance indicators to guide production [1, 2]. The meteorological factors of the corresponding stage are also different due to the difference of sowing time, and in the meanwhile react on the growth and development of maize. As a consequence, the changes of meteorological factors in each growth stage of maize can be adjusted by adjusting the sowing date so as to study the effects of meteorological factors such as light, temperature and water on the growth of maize [3, 4]. Sun et al. (1999) analyzed the effects of meteorological factors such as sunshine hours and photosynthetic radiation on the growth process of maize by the experiment of sowing by stages [5]. Besides, Li et al. (2002) showed that the accumulated temperature and sunshine hours during the growth stage of maize were significantly positively correlated with yield and yield components, and the sunshine hours in the flowering-maturity stage had a significant effect on the number of grains per spike. The daily average temperature difference was negatively correlated with accumulated 
temperature and had a negative effect on 1000-grain weight and yield [6]. Previous studies mainly focused on the effects of meteorological factors on grain yield and composition of common corn, but there were few studies on the effects of meteorological factors on fresh ear yield of fresh corn. In addition, there were differences among meteorological factors in different regions, which make them have different effects on crop yield. Based on these, we tried to analyze the effects of main meteorological factors on fresh ear yield of waxy maize in central Zhejiang. The whole growth stage of five waxy corn genotypes was divided into three growth stages: emergence stage, pre-flowering stage and filling stage, and the changes of meteorological factors in each corresponding stage were investigated. And then, the correlation between meteorological factors and fresh ear yield characters of waxy maize was statistically analyzed, and the effects of meteorological factors on yield characters were clarified. Finally, we tried to provide a basis for selecting suitable varieties and sowing date in production and putting forward matching cultivation techniques.

\section{Materials and Methods}

\subsection{Test Material and Design}

The experiment was carried out at the Zhejiang Experimental Station of the Institute of Maize and Featured Upland Crops, Zhejiang Academy of Agricultural Sciences, China $\left(29.16^{\circ} \mathrm{N}\right.$, $\left.120.13^{\circ} \mathrm{E}\right)$. The soil fertility of rice was uniform before the experiment. The split zone design was adopted in the experiment, the sowing date was the main treatment, and the variety was the secondary treatment. And the experiment was conducted from March to July 2017 and from March to July 2018, with three different sowing dates, with an interval of 15 days: early sowing dates (March 15), medium sowing dates (March 30) and late sowing dates (April 14) in 2017; early sowing dates (March 19), medium sowing dates (April 3) and late sowing dates (April 18) in 2018. The tested varieties were Huyunuo 3, Suyunuo 2, Meiyu 8, Zhe Fengnuo 3 and Jingkenuo 2000. Each variety had 6 rows per sowing date, the plant spacing was $0.25 \mathrm{~m}$, the row spacing was $0.75 \mathrm{~m}$, and the plot area was $36 \mathrm{~m}^{2}$. The yield was calculated in 4 rows in the middle of the plot, and protective rows were set around the experimental field. The fertilization method was as follows: $450 \mathrm{~kg} / \mathrm{hm}^{2}$ was applied in the ditch in the middle of the border before sowing, and covered with plastic film after burying and finishing. Besides, $150 \mathrm{~kg} / \mathrm{hm}^{2}$ was applied to the root at 5 leaves and 1 heart in the seedling stage, and urea $300 \mathrm{~kg} / \mathrm{hm}^{2}$ was applied to the interplant hole at the silking stage. What's more, the field pests were controlled uniformly, and other management measures were the same as the routine.

\subsection{Determination Content and Method}

We recorded the sowing time, emergence time and silking time of each treatment. It was recorded as the best harvest time for fresh ears when the filaments dried up and turned dark brown, the bracts showed slight signs of dehydration, and the waxy corn kernels were bright and full in color and milky paste flowed out after being pinched by hand by observing the appearance and grain characters [7]. And we confirmed that the emergence stage was from sowing time to emergence time, the pre-flowering stage was from emergence time to silking time, and the filling stage was from silking time to the best harvest time. The yield was calculated by collecting four rows of effective ears in the middle of the plot in the best harvest time, and converted into yield per hectare. Then we tried to select twenty representative ears to measure ear traits such as ear diameter, ear length, ear row number, kernels per row, bald tip length, 100-grain dry weight and so on.

\subsection{Meteorological Data}

The meteorological data of daily highest temperature, daily minimum temperature, daily average temperature, sunshine hours and daily rainfall during maize growth stage were provided by Meteorological Bureau of Dongyang city. Noting: The daily temperature difference was the difference between the daily maximum temperature and the daily minimum temperature; the number of sunshine hours was the sum of the daily average sunshine hours during the study period; the rainfall was the sum of the daily rainfall during the study period; the effective accumulated temperature was the sum of the daily effective temperature during the corn inspection period; the effective temperature was the difference of daily average temperature minus corn biological zero degrees (negative value was 0 ).

\subsection{Data Analysis}

We calculated data and drew charts with WPS2000 software, and conducted correlation and regression analysis with DPS7.05 software.

\section{Results and Analysis}

\subsection{Analysis on the Growth stage of Different Waxy Corn Varieties Under Different Sowing Dates}

It can be seen from figure 1 that in 2017, under the early sowing treatment, the emergence stage was 18-20 days, the pre-flowering stage was 63-70 days, the filling stage was 21-22 days; Under the middle sowing treatment, the emergence stage was 12-13 days, the pre-flowering stage was 57-64 days, the filling stage was 19-22 days; Under the late sowing treatment, the emergence stage was 6-9 days, the pre-flowering stage was 51-59 days, and the filling stage was 17-20 days. In the meanwhile, in 2018 , under the early sowing treatment, the emergence stage was 15-17 days, pre-flowering stage was 49-59 days, the filling stage was 20-22 days; Under the middle sowing treatment, the emergence stage was 10-11 days, the pre-flowering stage was 53-61 days, the filling stage was 21-23 days; Under the late sowing treatment, the emergence stage was 10-11 days, the pre-flowering stage was 48-60 days, the filling stage was 19-22 days. We could come to a conclusion that with the postponement of sowing time, the duration of each growth stage of each variety showed a trend of shortening. 


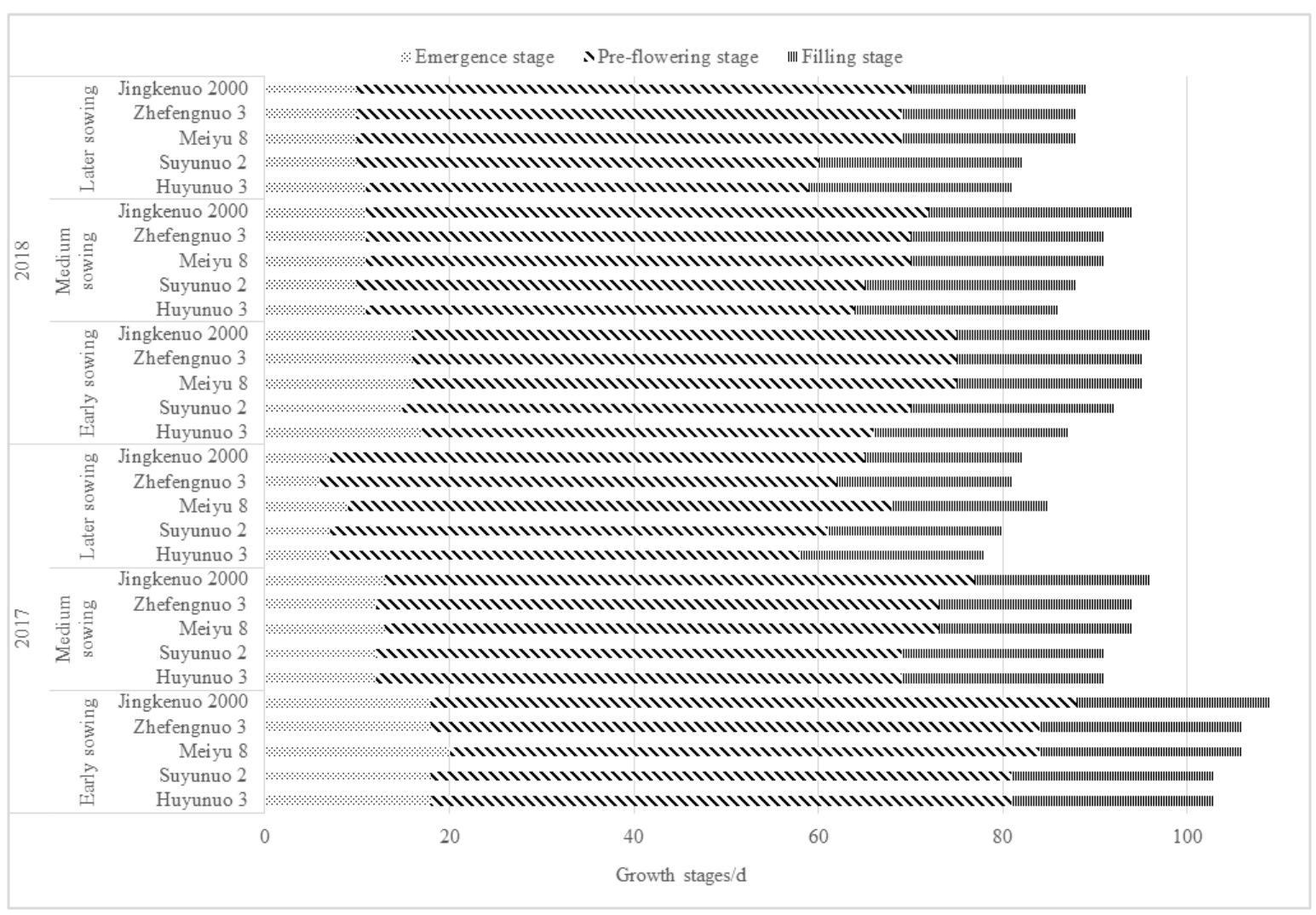

Figure 1. Growth stages of different waxy corn varieties under different sowing dates.

\subsection{Analysis of Meteorological Factors in Different Growth Stages of Corn Under Different Sowing Dates}

Table 1. Changes of the ecological factors in different planting dates.

\begin{tabular}{|c|c|c|c|c|c|c|c|c|c|c|c|}
\hline \multirow[b]{2}{*}{ growth stage } & \multirow[b]{2}{*}{ Meteorological factors } & \multicolumn{5}{|l|}{2017} & \multicolumn{5}{|l|}{2018} \\
\hline & & $\begin{array}{l}\text { Early } \\
\text { sowing }\end{array}$ & $\begin{array}{l}\text { Medium } \\
\text { sowing }\end{array}$ & $\begin{array}{l}\text { Later } \\
\text { sowing }\end{array}$ & Mean & CV (\%) & $\begin{array}{l}\text { Early } \\
\text { sowing }\end{array}$ & $\begin{array}{l}\text { Medium } \\
\text { sowing }\end{array}$ & $\begin{array}{l}\text { Later } \\
\text { sowing }\end{array}$ & Mean & CV (\%) \\
\hline \multirow{5}{*}{$\begin{array}{l}\text { Emergence } \\
\text { stage }\end{array}$} & Daily temperature difference $/{ }^{\circ} \mathrm{C}$ & 9.0 & 10.3 & 8.6 & 9.3 & 7.1 & 9.4 & 9.6 & 5.7 & 8.2 & 20.7 \\
\hline & Daily average temperature $/{ }^{\circ} \mathrm{C}$ & 13.9 & 15.6 & 18.2 & 15.9 & 9.4 & 16.5 & 18.3 & 17.5 & 17.5 & 3.6 \\
\hline & Rainfall/mm & 90.0 & 16.5 & 29.3 & 45.3 & 65.9 & 78.7 & 7.2 & 85.5 & 57.1 & 58.3 \\
\hline & Sunshine duration $/ \mathrm{h}$ & 62.8 & 77.6 & 25.7 & 55.3 & 35.8 & 62.7 & 55.4 & 16.4 & 44.8 & 42.3 \\
\hline & Effective accumulated temperature $/{ }^{\circ} \mathrm{C}$ & 78.8 & 75.3 & 66.6 & 73.6 & 6.3 & 110.8 & 98.0 & 84.4 & 97.7 & 9.1 \\
\hline \multirow{4}{*}{$\begin{array}{l}\text { Pre-flowerin } \\
\text { g stage }\end{array}$} & Daily average temperature $/{ }^{\circ} \mathrm{C}$ & 21.2 & 22.2 & 23.1 & 22.2 & 2.8 & 20.7 & 22.0 & 23.9 & 22.2 & 5.0 \\
\hline & Rainfall/mm & 365.1 & 387.6 & 396.3 & 383.0 & 3.1 & 345.7 & 361.7 & 449.7 & 385.7 & 11.1 \\
\hline & Sunshine duration $/ \mathrm{h}$ & 317.1 & 272.3 & 240.5 & 276.6 & 9.8 & 231.3 & 254.2 & 271.2 & 252.2 & 5.5 \\
\hline & Effective accumulated temperature $/{ }^{\circ} \mathrm{C}$ & 743.7 & 742.7 & 741.8 & 742.7 & 0.1 & 611.4 & 703.0 & 778.9 & 697.8 & 8.3 \\
\hline \multirow{4}{*}{ Filling stage } & Daily temperature difference $/{ }^{\circ} \mathrm{C}$ & 6.7 & 6.7 & 7.1 & 6.9 & 2.6 & 8.4 & 6.7 & 6.8 & 7.3 & 10.1 \\
\hline & Daily average temperature $/{ }^{\circ} \mathrm{C}$ & 26.6 & 27.2 & 28.5 & 27.4 & 2.6 & 26.2 & 25.4 & 26.7 & 26.1 & 1.7 \\
\hline & Rainfall/mm & 155.1 & 150.0 & 107.2 & 137.4 & 14.7 & 137.3 & 333.7 & 242.5 & 237.8 & 28.2 \\
\hline & Sunshine duration $/ \mathrm{h}$ & 61.5 & 64.7 & 79.8 & 68.7 & 10.8 & 126.5 & 71.7 & 67.1 & 88.4 & 28.7 \\
\hline
\end{tabular}

Combined with figure 1, we tried to conduct statistics on the meteorological factors of each corresponding growth stage. As shown in Table 1, the daily average temperature of the emergence stage varies greatly under different sowing dates in 2017 with a coefficient of variation of $9.4 \%$. It might be the reason for the great difference in emergence stage among different sowing dates, indicating that temperature was the most important factor in the emergence speed of maize seedlings under the condition of sufficient water. Except for the great difference of sunshine duration at different sowing dates in 2017, the variation range of other meteorological factors was relatively small. In 2018, except for the small daily temperature difference, the coefficient of variation of other meteorological factors reached more than $5 \%$. The variation of daily temperature difference in filling stage was small, and the daily average temperature increased with the postponement of sowing date under the treatment of sowing date in 2017. Besides, in 2018, the daily temperature 
difference in the filling stage of different sowing dates varied greatly, and the average daily temperature decreased at first and then increased with the postponement of sowing date. The reason for this may be the rainy weather during the filling stage. During the period, the cumulative precipitation reached $333.7 \mathrm{~mm}$, which was the highest among all sowing dates.

\subsection{Correlation Analysis Between Meteorological Factors and Maize Yield Characters in Different Corn Growth Stages}

Table 2. Correlation between meteorological factors at different growth stages and yield traits of Maize.

\begin{tabular}{|c|c|c|c|c|c|c|c|c|}
\hline growth stages & Meteorological factors & Ear length & $\begin{array}{l}\text { Ear } \\
\text { diameter }\end{array}$ & $\begin{array}{l}\text { Ear row } \\
\text { number }\end{array}$ & $\begin{array}{l}\text { Grain number } \\
\text { per row }\end{array}$ & $\begin{array}{l}\text { Bald tip } \\
\text { length }\end{array}$ & $\begin{array}{l}\text { 100-grain } \\
\text { dry weight }\end{array}$ & Yield \\
\hline \multirow{5}{*}{ Emergence stage } & Daily temperature difference & 0.18 & 0.13 & 0.04 & 0.19 & -0.06 & 0.16 & 0.32 \\
\hline & Daily average temperature & -0.08 & -0.24 & -0.01 & -0.11 & 0.24 & -0.26 & -0.33 \\
\hline & Rainfall & 0.05 & 0.10 & -0.02 & 0.13 & -0.20 & 0.09 & 0.12 \\
\hline & Sunshine duration & 0.21 & 0.08 & 0.06 & 0.23 & -0.02 & 0.10 & 0.25 \\
\hline & Effective accumulated temperature & 0.15 & -0.15 & 0.02 & 0.24 & 0.13 & -0.20 & -0.10 \\
\hline \multirow{4}{*}{$\begin{array}{l}\text { Pre-flowering } \\
\text { stage }\end{array}$} & Daily temperature difference & -0.07 & 0.14 & 0.16 & -0.15 & -0.25 & 0.21 & 0.22 \\
\hline & Rainfall & 0.23 & -0.06 & $-0.37 *$ & 0.28 & 0.28 & -0.13 & -0.15 \\
\hline & Sunshine duration & 0.14 & -0.13 & -0.25 & 0.01 & 0.30 & -0.15 & -0.18 \\
\hline & Effective accumulated temperature & 0.27 & -0.20 & $-0.42 *$ & 0.13 & $0.53 * *$ & -0.27 & -0.32 \\
\hline \multirow{4}{*}{ Filling stage } & Daily temperature difference & 0.16 & $0.39 *$ & 0.01 & $0.46 * *$ & $-0.39 *$ & $0.37 *$ & $0.51 * *$ \\
\hline & Daily average temperature & 0.26 & 0.29 & -0.17 & 0.31 & -0.09 & 0.29 & 0.33 \\
\hline & Rainfall & -0.26 & $-0.43 *$ & 0.00 & $-0.39 *$ & $0.49 * *$ & $-0.46 * *$ & $-0.56 * *$ \\
\hline & Sunshine duration & 0.15 & 0.35 & -0.05 & $0.43^{*}$ & -0.30 & 0.31 & $0.48 * *$ \\
\hline
\end{tabular}

We could know from Table 2 that the meteorological factors of each growth stage had no significant effect on ear length; the ear row number was significantly negatively correlated with the effective accumulated temperature and rainfall in the early flowering stage, and the ear diameter was positively correlated with the daily temperature difference at the filling stage, negatively correlated with the rainfall at the filling stage, and positively correlated with the effective accumulated temperature at the filling stage; The grain number per row has a very significant positive correlation with the daily temperature difference during the filling stage, a significant positive correlation with the sunshine hours during the filling stage, and a significant negative correlation with the rainfall during the filling stage; The bald tip of corn was significantly positively correlated with the effective accumulated temperature at the pre-flowering stage, positively correlated with the precipitation during the filling stage, negatively correlated with the daily temperature difference during the filling stage, and negatively correlated with the effective accumulated temperature during the filling stage. The results showed that increasing the average daily temperature difference, reducing precipitation and increasing effective accumulated temperature during the filling stage could effectively reduce the formation of corn bald tip. Besides, there was a significant positive correlation between 100 -grain weight and daily temperature difference during the filling stage, an extremely significant positive correlation between 100-grain weight and effective accumulated temperature during the filling stage, and an extremely significant negative correlation between 100-grain weight and rainfall during filling stage. It shows that increasing the daily temperature difference and effective accumulated temperature and reducing rainfall are beneficial to the increase of 100-grain weight. What's more, the yield was significantly negatively correlated with the daily average temperature during the pre-flowering stage, significantly positively correlated with the daily temperature difference, sunshine hours and effective accumulated temperature during the filling stage, and negatively correlated with the precipitation during the filling stage, indicating that reducing the daily average temperature during the pre-flowering stage and reducing the precipitation during the filling stage contributed to the increase of yield by increasing the daily temperature difference, sunshine hours and effective accumulated temperature during the filling stage.

\subsection{Yield of Different Waxy Corn Varieties Under Different Sowing Dates}

Table 3. Yield of different maize varieties under different sowing dates.

\begin{tabular}{|c|c|c|c|c|c|c|c|c|c|c|}
\hline \multirow[b]{2}{*}{ varieties } & \multicolumn{5}{|c|}{$2017\left(\mathrm{~kg} / \mathrm{hm}^{2}\right)$} & \multicolumn{5}{|c|}{$2018\left(\mathrm{~kg} / \mathrm{hm}^{2}\right)$} \\
\hline & $\begin{array}{l}\text { Early } \\
\text { sowing }\end{array}$ & $\begin{array}{l}\text { Medium } \\
\text { sowing }\end{array}$ & $\begin{array}{l}\text { Later } \\
\text { sowing }\end{array}$ & Mean & CV (\%) & $\begin{array}{l}\text { Early } \\
\text { sowing }\end{array}$ & $\begin{array}{l}\text { Medium } \\
\text { sowing }\end{array}$ & $\begin{array}{l}\text { Later } \\
\text { sowing }\end{array}$ & Mean & CV (\%) \\
\hline Huyunuo 3 & 15252.9 & 14869.1 & 14758.1 & 14960.0 & 1.3 & 15558.4 & 14533.3 & 13684.9 & 14592.2 & 4.4 \\
\hline Suyunuo 2 & 15669.5 & 15285.7 & 15630.5 & 15528.6 & 1.0 & 16983.5 & 15029.7 & 14172.4 & 15395.2 & 6.9 \\
\hline Meiyu 8 & 14267.5 & 14766.1 & 14526.8 & 14520.2 & 1.2 & 14739.9 & 12498.7 & 13540.1 & 13592.9 & 5.6 \\
\hline Zhefengnuo 3 & 13988.2 & 14520.1 & 15074.9 & 14527.7 & 2.5 & 14489.9 & 12248.7 & 13290.1 & 13342.9 & 5.7 \\
\hline Jingkenuo 2000 & 15814.5 & 16094.4 & 15327.4 & 15745.4 & 1.8 & 16082.1 & 14383.5 & 14664.5 & 15043.4 & 4.6 \\
\hline $\mathrm{CV}(\%)$ & 4.6 & 3.1 & 2.2 & & & 4.9 & 7.9 & 3.2 & & \\
\hline
\end{tabular}


As we can see from the table 3 , the yield variation coefficient of each sowing period in 2017 was small, the yield of middle sowing treatment was the highest, slightly higher than that of early sowing treatment, and the yield of late sowing treatment was the lowest. Besides, the yield varied greatly during each sowing period in 2018. The yield of early sowing treatment was the highest, followed by late sowing, and the middle sowing treatment was the lowest, slightly lower than late sowing. In 2017, the fresh ear yield of Jingkenuo 2000 was the highest, followed by Suyunuo 2, Huyunuo 3, Meiyu 8 and Zhefengnuo 3. At the same time, in 2018, the fresh ear yield of Suyunuo 2 was the highest, followed by Jingkenuo 2000, Huyunuo 3, Meiyu 8 and Zhefengnuo 3.

\subsection{Regression Analysis of Meteorological Factors on Fresh Ear Yield of Corn}

Many studies showed that the yield formation of maize was greatly affected by meteorological factors during filling stage [8, 9]. In this study, we tried to make linear regression analysis between the main meteorological factors and fresh ear yield of waxy corn during filling stage, in order to understand the effect of meteorological factors on yield. As shown in figure 2, the relationship between the five main meteorological factors and the yield conformed to the quadratic curve and had a significant regression relationship. There was an increasing relationship between yield and daily temperature difference, sunshine hours and effective accumulated temperature through mapping and analysis. Besides, the yield increased first and then decreased with the average daily temperature, which showed that proper temperature increase could promote the yield, but too high temperature was disadvantageous to the yield. However, there was a decreasing relationship between yield and rainfall, which was inconsistent with the study of Li et al. (2012) that rainfall promoted the increase of yield [10]. The reason may be that the grain filling stage of maize in central Zhejiang often overlapped with the Meiyu period, leading to the large precipitation; Besides, there was basically no drought in the soil, and too much Rain Water was not conducive to the formation of yield; What's more, too much Rain Water would weak the daily temperature difference and sunshine hours and other useful meteorological factors on the yield, and indirectly affected the yield.

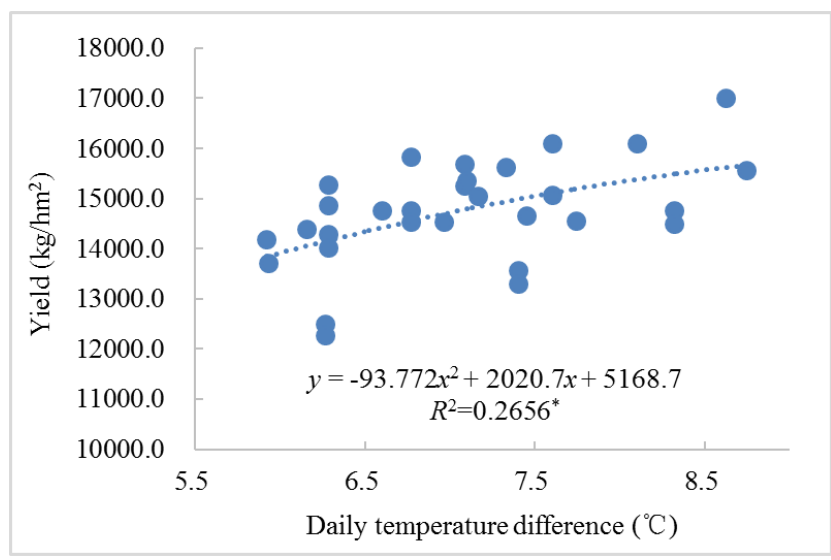

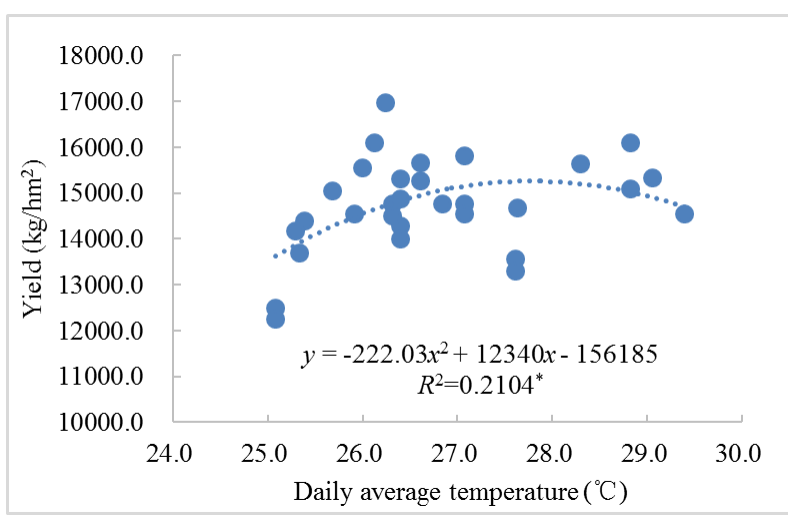
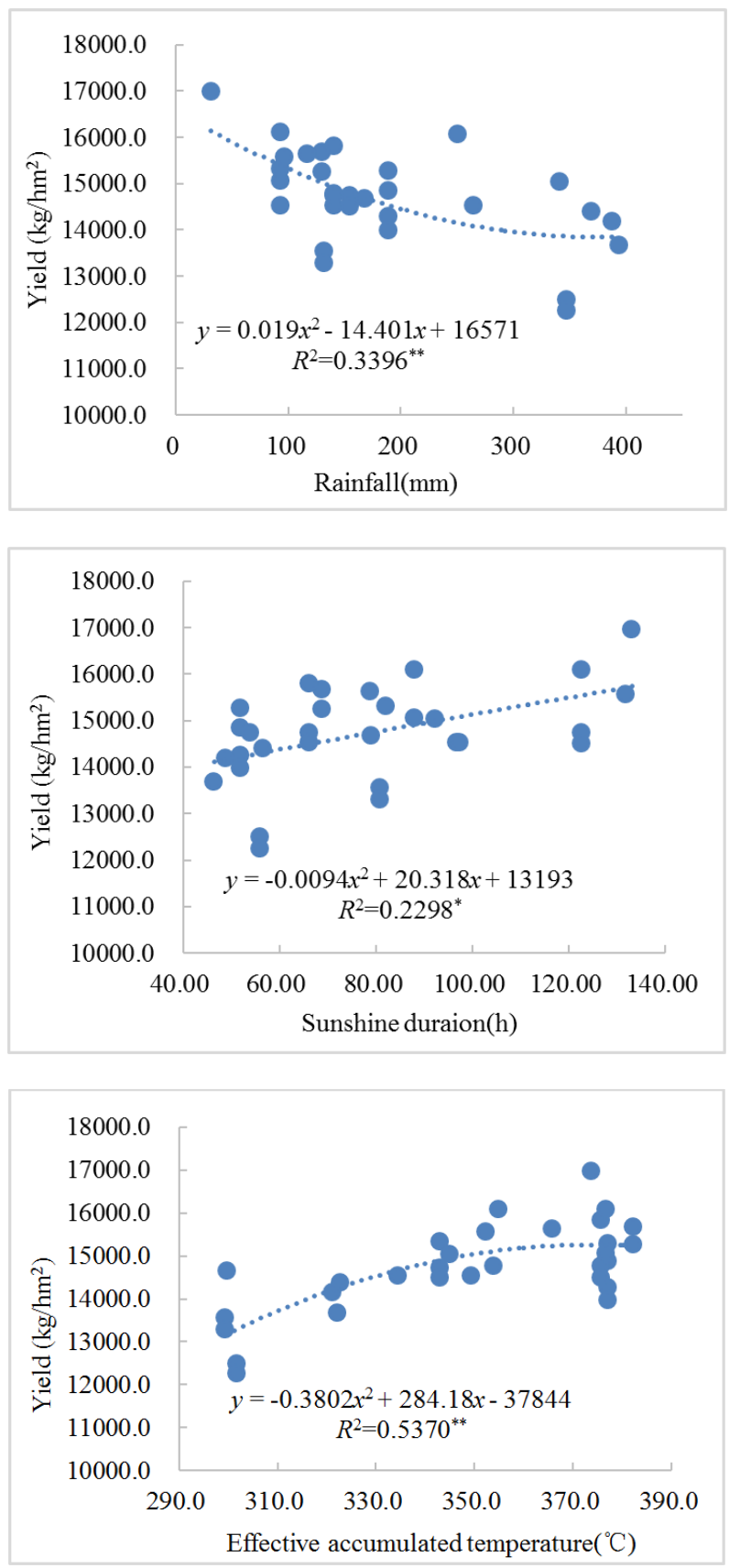

Figure 2. Regression analysis of meteorological factors in the filling stage on fresh ear yield. 


\section{Discussion}

Tao et al. (2013) believed that high temperature during the filling stage of corn was easy to produce high temperature stress on corn [11]. The further increase of temperature would adversely affect the yield when the overall temperature of corn is too high during the filling stage. In our study, increasing temperature at filling stage had a positive effect on yield formation, but too high temperature would force corn to mature and shorten filling time. The result was similar to the study of Wang et al. (2015) that too high or too low temperature during the filling stage was not conducive to the accumulation and transportation of dry matter in corn [12]. There was a very significant increasing relationship between effective accumulated temperature during filling stage and fresh ear yield of corn, which showed that increasing effective accumulated temperature during filling stage was beneficial to yield increase. It is more beneficial to increase the effective accumulated temperature by properly reducing the temperature during the filling stage to prolong the filling time because the effective accumulated temperature was affected by temperature and time, which could further increase the 100-grain weight and fresh ear yield of corn. The results was similar to the results of studies that early sowing at the right time could prolong the growth stage of corn, increase the effective accumulated temperature, and increase plant dry matter and grain yield [13-15]. In addition, Rain Water was sufficient in the filling stage of spring corn in central Zhejiang, which was basically not affected by drought. Too much Rain Water would reduce the daily temperature difference, sunshine hours and other meteorological factors beneficial to the growth of maize, which was disadvantageous to the yield. Lu et al. (2011) showed that the sowing date significantly affected the distribution of waxy corn starch grains, and the reason for the larger starch grains of waxy corn under spring sowing treatment was related to high temperature, Meiyu and low light in the later stage [16]. We could know that high temperature, Meiyu and low light in the later stage also have effects on the quality of waxy corn. As a consequence, the extremely high temperature and rainy weather should be avoided during the grain filling stage of corn production.

The growth stage of corn changed at different stages, and the corresponding meteorological factors also changed under the double influence of different sowing dates and varieties. The variety Suyunuo 2 not only had high yield, but also grew early, and had the advantage of high yield under the condition of early sowing through analysis. Besides, the yield of Jingkenuo 2000 was higher, but the growth stage was longer, and the yield of late sowing was greatly affected by adverse meteorological factors in the later stage. What's more, the overall yield of early sowing in 2017 was slightly lower than that of middle and late sowing, which might be related to the overall seedling emergence in the early stage. Early sowing in central Zhejiang before mid-March, the temperature might not reach the effective temperature for maize growth, and the seeds were easy to rot or sprout slowly after sowing. Thus it prolonged the whole emergence time, and it was easy to cause lack of seedlings or large and small seedlings of corn. At last, it affected the formation of late yield. It was suggested that plastic film mulching should be used to improve the water and heat condition of soil and increase the emergence rate and uniformity of corn [17]. The overall yield of each variety was low due to the influence of continuous overcast and rainy weather during grain filling under the condition of middle sowing and late sowing in 2018. Besides, the sowing temperature was stable after the middle of April, but it might lead to the high temperature during the filling stage, which might make the corn mature too quickly, shorten the filling time, reduce the effective accumulated temperature, and easily cause insufficient filling or bald point. Finally, it had an adverse effect on fresh ear yield and marketability. In our study, we considered that the average temperature of maize planting in spring should be stable above $12^{\circ} \mathrm{C}$. Besides, we suggested that direct seeding of corn field should be carried out in the middle of Zhejiang Province after the middle of March and before the middle of April. In the meanwhile, we should combine with the local weather forecast to determine the sowing date. Li et al. (2013) showed that early sowing along the Huaihe River would lead to the phenomenon of too low temperature during the filling stage, which was not conducive to grain filling and yield formation [18]. However, the temperature conditions in mid-early May still meet the normal needs of maize grain filling according to the analysis of meteorological conditions in central Zhejiang over the years. As a consequence, we confirmed that it could be sowed early in central Zhejiang without affecting seed emergence, and the conditional areas could also use greenhouse seedlings from the end of February to the beginning of March. And then we transplant in the field in the middle and late March. Besides, using plastic film warming to increase the temperature demand of maize in the early stage, which can not only put corn on the market earlier, but also leave enough growth time for the crops after the double cropping system [19].

Waxy corn originated in China and is one of the important food and economic crops. It is of great significance to study the relationship between meteorological factors and yield in order to promote the production of waxy corn. However, the purpose of this experiment is only to study the influence of meteorological factors on the yield of fresh ear of waxy corn. The influence degree of diseases and insect pests and the change of internal quality of waxy corn under different environmental conditions are also worth studying. The effects of different meteorological factors on diseases and insect pests and grain quality of waxy corn need to be analyzed by follow-up experiments.

\section{Conclusion}

Spring corn planting in central Zhejiang can be sowed early appropriately under the condition that the sowing temperature is normal and maize emergence is not affected, which can move forward the whole growing stage of corn. Besides, it can weaken the effects of high temperature and rainy weather 
during corn filling, prolong the grain filling time, increase the effective accumulated temperature, and form a favorable climatic condition for maize growth as a whole. Finally, it contributes to improving the commodity of corn ear and the yield of fresh ear.

\section{Acknowledgements}

This work was supported by the China Agriculture Research System on Maize Industry (grant No. CARS-02-69), Major Agriculture Science Foundation of Upland Grain Crops Breeding of Zhejiang Province (grant No. 2016C02050-9-1), and the national key research and development program of China (grant No. 2018YFD0200706).

\section{References}

[1] Hou Y H, Chen C Y, Guo Z Q, et al. Dynamic characteristics of leaf area index and allocation characteristics of ecological resources for different yielding spring maize populations. Chinese Journal of Applied Ecology, 2009, 20 (1): 135-142. (in Chinese).

[2] Zhang B, Zhao M, Dong Z Q, et al. Establishment and test of LAI dynamic simulation model for high yield population. Acta Agronomica Sinica, 2007, 33 (10): 1674-1688. (in Chinese).

[3] Dong F, Yan Q Y, Li F, et al. Effects of sowing date and planting density on growth and yield of dry-land maize. Journal of Maize Sciences, 2020, 28 (2): 115-121. (in Chinese).

[4] Han H M, Zhang L, Sun M, et al. Response of growth, development and yield of different summer maize cultivars to sowing date in Huang-Huai-Hai plain. Journal of Maize Sciences, 2020, 28 (2); 106-114. (in Chinese).

[5] Sun Y T, Sun M M. The assessment model of temperature on maize growth and development. Resource Sciences, 1999, 34 (1): 63-70. (in Chinese).

[6] Li Y Z, Dong X W, Liu G L, et al. Effect of light and temperature factor on yield and its components in maize. Chinese Journal of Eco-Agriculture, 2002, 10 (2): 86-89. (in Chinese).

[7] Sun R, Wang G Q, Zhuang Y. Progress on harvesting time and physiological characteristics of post-harvest fresh ears of waxy corn. Journal of Tianjin Agricultural University, 2014, 3 (21): 43-45. (in Chinese).

[8] Guo Y Q, Guo X Y, Zhao C J, et al. Chun-jiang Scientia Agricultura Sinica Design and Implementation of a Dynamic Knowledge Model for Suitable Cultivars and Sowing Time of
Maize. Scientia Agricultura Sinica, 2006, 39 (2): 274-280. (in Chinese).

[9] Zhang B S, Li S Y, Hu L H, et al. The effects of sowing season on growth, development, dry matter production and partition of maize (Zea mays). Acta Botanica Yunnanica, 2001, 23 (2): 243-250. (in Chinese).

[10] Li X L, Li C F, Hou Y H, et al. Dynamic characteristics of summer maize yield performance in different planting dates and its effect of ecological factors. Scientia Agricultura Sinica, 2012, 45 (6): 1074-1083. (in Chinese).

[11] Tao Z Q, Chen Y Q, Li C, et al. Path nalysis between yield of spring maize and meteorological factors at different sowing times in North China low plain. Acta agronomica sinica, 2013, 39 (9): 1628-1634. (in Chinese).

[12] Wang X Q, Yang Y L, Zhang Y, et al. Bulletin analysis of the effects of meteorological factors at different growth stages on yield and yield components of maize (Zea mays L.). Chinese Agricultural Science, 2015, 31 (30): 68-73. (in Chinese).

[13] Liu Y E, Xie R Z, Zhang H B, et al. Study on increasing rate of maize yield after putting off harvest time in different ecoregions. Scientia Agricultura Sinica, 2010, 43 (13): 2820-2828. (in Chinese).

[14] Li C H, Su X H, Xie R Z, et al. Study on relationship between grain-yield of summer corn and climatic ecological condition (in Chinese) under super-high-yield cultivation. Scientia Agriculture Sinica, 2001, 34 (3): 311-316. (in Chinese).

[15] Wang G Y, Zhao F C, Bao F, et al. Study on grain filling characteristics of waxy maize hybrids with different sowing dates. Molecular Plant Breeding, 2017, 15 (9): 3811-3. (in Chinese).

[16] Lu D L, Guo H F, Lu W P. Effects of Sowing Date, Variety and nitrogen top-dressing at jointing stage on starch granule size distribution of waxy maize. Scientia Agricultura Sinica, 2011, 44 (2): 263-270. (in Chinese).

[17] Zheng H J, Dong S T, Wang K J, et al. Effects of ecological factors on maize (Zea mays L.) yield of different varieties and corresponding regulative measure. Acta Agronomica Sinica, 2001, 27 (6): 863-867. (in Chinese).

[18] Li W Y, Wang C J, Zhang Z X, et al. The relationship between grain filling characteristics of summer maize and main meteorological factors during grain filling under different planting date. Journal of Anhui Science and Technology University, 2013, 27 (5): 21-26. (in Chinese).

[19] Zhang Z T, Yang X G, Gao J Q, et al. Analysis of suitable sowing date for summer maize in North China plain under climate change. Scientia Agricultura Sinica, 2018, 51 (17): 3258-3274. (in Chinese). 